\title{
The fallacy of enrolling only high-risk subjects in cancer prevention trials: Is there a "free lunch"? Stuart G Baker*1, Barnett S Kramer ${ }^{2}$ and Donald Corle ${ }^{1}$
}

Address: ${ }^{1}$ Biometry Research Group, Division of Cancer Prevention, National Cancer Institute, Bethesda, Maryland, USA and ${ }^{2}$ Office of Disease Prevention, National Institutes of Health, Bethesda, Maryland, USA

Email: Stuart G Baker* - sb16i@nih.gov; Barnett S Kramer - KramerB@OD.NIH.GOV; Donald Corle - dc13a@nih.gov

* Corresponding author

Published: 04 October 2004

BMC Medical Research Methodology 2004, 4:24 doi:I0.I I86/I47|-2288-4-24

This article is available from: http://www.biomedcentral.com/147I-2288/4/24

(C) 2004 Baker et al; licensee BioMed Central Ltd.

This is an open-access article distributed under the terms of the Creative Commons Attribution License (http://creativecommons.org/licenses/by/2.0), which permits unrestricted use, distribution, and reproduction in any medium, provided the original work is properly cited.

\begin{abstract}
Background: There is a common belief that most cancer prevention trials should be restricted to highrisk subjects in order to increase statistical power. This strategy is appropriate if the ultimate target population is subjects at the same high-risk. However if the target population is the general population, three assumptions may underlie the decision to enroll high-risk subject instead of average-risk subjects from the general population: higher statistical power for the same sample size, lower costs for the same power and type I error, and a correct ratio of benefits to harms. We critically investigate the plausibility of these assumptions.

Methods: We considered each assumption in the context of a simple example. We investigated statistical power for fixed sample size when the investigators assume that relative risk is invariant over risk group, but when, in reality, risk difference is invariant over risk groups. We investigated possible costs when a trial of high-risk subjects has the same power and type I error as a larger trial of average-risk subjects from the general population. We investigated the ratios of benefit to harms when extrapolating from high-risk to average-risk subjects.

Results: Appearances here are misleading. First, the increase in statistical power with a trial of high-risk subjects rather than the same number of average-risk subjects from the general population assumes that the relative risk is the same for high-risk and average-risk subjects. However, if the absolute risk difference rather than the relative risk were the same, the power can be less with the high-risk subjects. In the analysis of data from a cancer prevention trial, we found that invariance of absolute risk difference over risk groups was nearly as plausible as invariance of relative risk over risk groups. Therefore a priori assumptions of constant relative risk across risk groups are not robust, limiting extrapolation of estimates of benefit to the general population. Second, a trial of high-risk subjects may cost more than a larger trial of average risk subjects with the same power and type I error because of additional recruitment and diagnostic testing to identify high-risk subjects. Third, the ratio of benefits to harms may be more favorable in high-risk persons than in average-risk persons in the general population, which means that extrapolating this ratio to the general population would be misleading. Thus there is no free lunch when using a trial of high-risk subjects to extrapolate results to the general population.
\end{abstract}

Conclusion: Unless the intervention is targeted to only high-risk subjects, cancer prevention trials should be implemented in the general population. 


\section{Background}

Some prevention trials are restricted to high-risk subjects. If the investigators are only interested in the effects of the intervention on subjects at increased risk [1] or if the study is designed to be a preliminary investigation in preparation for a definitive study in the general population, we think this restriction is reasonable.

However some investigators who are interested in studying the effect of the intervention in the general population may be tempted to design a "definitive" study to estimate the effect of the intervention in a high-risk group. Some investigators may believe that a trial of high-risk subjects would have greater power than a trial of the same size among average-risk subjects. Some examples of this type of thinking can be found in papers on risk prediction models $[2,3]$. Some investigators may believe that a trial of high-risk subjects with the same power as a trial of average-risk subjects would have lower costs than a trial of average-risk subjects. Some investigators may believe the ratio of benefits to harms can be correctly extrapolated from high-risk to average-risk subjects. Although the rationales for these various beliefs are related, they involve some distinct underlying assumptions that are important to critically examine.

\section{Methods and results \\ Possibly lower statistical power}

To crystallize our thinking about statistical power, we consider the following simple hypothetical and realistic example. Investigators want to estimate the effect of intervention in the general population, so they first consider designing a randomized trial among the general at-risk population. Suppose they anticipate that the cumulative probability of incident cancer over the course of the study is $p_{C}=.02$ in the control arm and $p_{I}=.01$ in the study arm, and they believe that the difference in probabilities is clinically significant. Also suppose that due to the limited availability of the intervention, they can enroll at most $n$ $=2000$ study participants in each arm. The investigators compute power using the following standard formula [1] setting the two-sided type I error at .05,

$$
\text { power }=\operatorname{NormalCDF}\left(\left(\Delta \sqrt{n}-1.96 s e_{N u l l}\right) / s e_{A l t}\right),
$$

where NormalCDF is the cumulative distribution function for a normal distribution with mean 0 and variance $1, \Delta$ is the anticipated difference one wants to detect, $n$ is the sample size per arm, $s e_{\text {Null }}$ is the standard error under the null hypothesis, and $s e_{A l t}$ is the standard error under the alternative hypothesis. Let $p=\left(p_{C}+p_{I}\right) / 2$. As discussed in [1], for a study designed to estimate the absolute risk difference, the statistic of interest is $\hat{p}_{C}-\hat{p}_{I}$, so

$$
\begin{aligned}
& \Delta=p_{C}-p_{I}, \\
& s e_{N u l l}=\sqrt{2 p(1-p),} \\
& s e_{\text {Alt }}=\sqrt{p_{C}\left(1-p_{C}\right)+p_{I}\left(1-p_{I}\right)} .
\end{aligned}
$$

For a study designed to estimate the relative risk, the statistic of interest is $\log \left(p_{C}\right)-\log \left(p_{I}\right)$, so

$$
\begin{aligned}
& \Delta=\log \left(p_{C}\right)-\log \left(p_{I}\right), \\
& s e_{N u l l}=\sqrt{2(1-p) / p} \\
& s e_{A l t}=\sqrt{\left(1-p_{C}\right) / p_{C}+\left(1-p_{I}\right) / p_{I}} .
\end{aligned}
$$

Applying these formulas to the above example and substituting either (2) or (3) into (1), the investigators obtain a power of .74 based on the absolute risk difference statistic and a power .76 based on a relative risk statistic [see Additional file 1].

Suppose the investigators think this power is too low. To increase power they propose to restrict the study to a highrisk group in which the probability of cancer is .04. Also suppose the investigators make the typical assumption that if the intervention yields a relative risk of .5 in the general population, it would also yield a relative risk of .5 in the high-risk group. Applying (1-3) with high risk subjects for whom $p_{C}=.04$ and $p_{I}=.02$ with $n=2000$, the investigators compute a power of .96 using either the absolute risk difference or relative risk. Because the power is higher using high-risk subjects, the investigators plan the study for a high-risk population and will generalize the results to the general population.

Is there a free lunch? An underlying assumption in this example is that the relative risk is invariant between the general population and the high-risk group. There is no free lunch because the impact of violating this assumption could be substantial. For example, suppose instead that the absolute risk difference is invariant between the general population and the high risk group. Under this scenario the absolute risk difference in the general population is .01 , so the absolute risk difference in the high-risk group is also .01. In this case for $p_{C}=.04, p_{I}=.03$, and $n=2000$, the power (computed using either absolute risk difference or relative risk statistics) for the trial of high-risk subjects is only .41. The decreased power in a high risk group under a constant risk difference model is not surprising: if the risk difference $p_{C}-p_{I}$ is the same, but $p_{I}$ is increasing, the variances, $p_{C}\left(1-p_{C}\right) / n$ and $p_{I}\left(1-p_{I}\right) / n$, will increase as $p_{C}$ increases up to .5 , which will reduce the power.

A crucial issue is whether or not the absolute risk difference or the relative risk is likely invariant between average- 
Table I: Data from a cancer prevention trial for investigating assumptions of constant risk difference and relative risk when risk groups change.

\begin{tabular}{|c|c|c|c|c|c|c|}
\hline \multirow[b]{2}{*}{ Variable } & \multirow[b]{2}{*}{ risk group } & & \multicolumn{2}{|c|}{ Placebo group } & \multicolumn{2}{|c|}{ Tamoxifen group } \\
\hline & & & cancer & at risk & cancer & at risk \\
\hline \multirow[t]{3}{*}{ age at entry } & 1 & $\leq 49$ & 68 & 10149 & 38 & 10045 \\
\hline & 2 & $50-59$ & 50 & 7912 & 25 & 8040 \\
\hline & 3 & $>60$ & 57 & 7719 & 26 & 7782 \\
\hline \multirow[t]{4}{*}{ predicted risk } & 1 & $\leq 2.00 \%$ & 35 & 6318 & 13 & 6311 \\
\hline & 2 & $2.01-3.01 \%$ & 42 & 8108 & 29 & 8262 \\
\hline & 3 & $3.01-5.00 \%$ & 43 & 7313 & 27 & 6959 \\
\hline & 4 & $\leq 5.01 \%$ & 55 & 4142 & 20 & 4425 \\
\hline \multirow[t]{4}{*}{ family risk } & 1 & 0 & 38 & 5891 & 17 & 5724 \\
\hline & 2 & I & 90 & 15000 & 46 & 15182 \\
\hline & 3 & 2 & 37 & 4263 & 20 & 4211 \\
\hline & 4 & 3 & 10 & 729 & 6 & 855 \\
\hline
\end{tabular}

Cancer is invasive breast cancer. Predicted risk is the 5 -year predicted risk. Family risk is number of first degree relatives with breast cancer. Data are from Table 5 of [5] with number at risk computed by dividing number of breast cancers by reported breast cancer rate.

risk subjects in the general population and high-risk subjects. The answer depends on the cancer, the interventions, and the biology. To gain some appreciation of this issue, we analyzed published data (summarized in Table 1) from a prevention trial of particular interest to us, a study of tamoxifen for the prevention of breast cancer [5]. Rather than limit the analysis to one particular high-risk group, we investigated subjects at various levels of risk defined separately by three variables: age, predicted risk, (the five-year risk of cancer based on the Gail model [3]), and family risk. We fit four models separately to each variable:

constant risk difference,

Constant $R D . \quad \operatorname{pr}($ invasive breast cancer $\mid$ placebo, group $i)=\pi_{i}$, $\operatorname{pr}$ (invasive breast cancer $\mid$ tamoxifen, group $i)=\pi_{i}-\delta$,

where $\delta$ is the risk difference that is constant over groups;

varying risk difference,

Varying $R D: \quad \operatorname{pr}($ invasive breast cancer $\mid$ placebo, group $i)=\pi_{i}$, $\operatorname{pr}($ invasive breast cancer $\mid$ tamoxifen, group $i)=\pi_{i}-\delta_{i}$,

where $\delta_{i}$ is the risk difference that varies over groups;

constant relative risk,

Constant RR: $\quad \operatorname{pr}($ invasive breast cancer $\mid$ placebo, group $i)=\pi_{i}$ $\operatorname{pr}($ invasive breast cancer $\mid$ tamoxifen, group $i)=\pi_{i} / \beta$,

where $\beta$ is the relative risk that is constant over groups;

varying relative risk, $\begin{array}{ll}\text { Varying RR: } & \operatorname{pr}(\text { invasive breast cancer } \mid \text { placebo, group } i)=\pi_{i}, \\ & \operatorname{pr}(\text { invasive breast cancer } \mid \text { tamoxifen, group } i)=\pi_{i} / \beta_{i},\end{array}$

where $\beta$ is the relative risk that varies over groups.

We obtained maximum likelihood estimates of $\delta, \delta_{i}, \beta$, and $\beta_{i}$ using a Newton-Raphson procedure [see Additional file 2].

To investigate the plausibility of the constant relative risk and constant risk difference models in this example, we plotted the estimates of $\delta, \delta_{i}, \beta$, and $\beta_{i}$ along with confidence intervals (Figure 1). In the top row of Figure 1 we plotted points corresponding to $\hat{\delta}_{j}$ with $(100-5 / k) \%$ confidence intervals and horizontal lines for $\hat{\delta}$ with $95 \%$ confidence intervals. We also presented the p-values corresponding to twice the difference in log-likelihoods for Varying $R D$ versus Constant $R D$. Similarly, in the bottom row of Figure 1, we plotted points corresponding to $\hat{\beta}_{j}$ with $(100-5 / k) \%$ confidence intervals and horizontal lines for $\hat{\beta}$ with $95 \%$ confidence intervals. We also presented the p-value corresponding to twice the difference in log-likelihoods for Varying $R R$ versus Constant $R R$. Out of 6 p-values ( 3 risk factors $\times 2$ statistics) only one, for absolute risk difference under the risk factor of predicted risk had a small p-value (and the p-value of .01 would not be significant at the .05 level under a Bonferroni adjustment of .05/6). Based on these p-values and inspection of Figure 1, the models Constant $R D$ and Constant RR are both plausible, especially for age and family risk.

The trial designer does not know the true state of nature. If Constant RD is the true state of nature, the power will be lower in the high-risk group than the general population. 


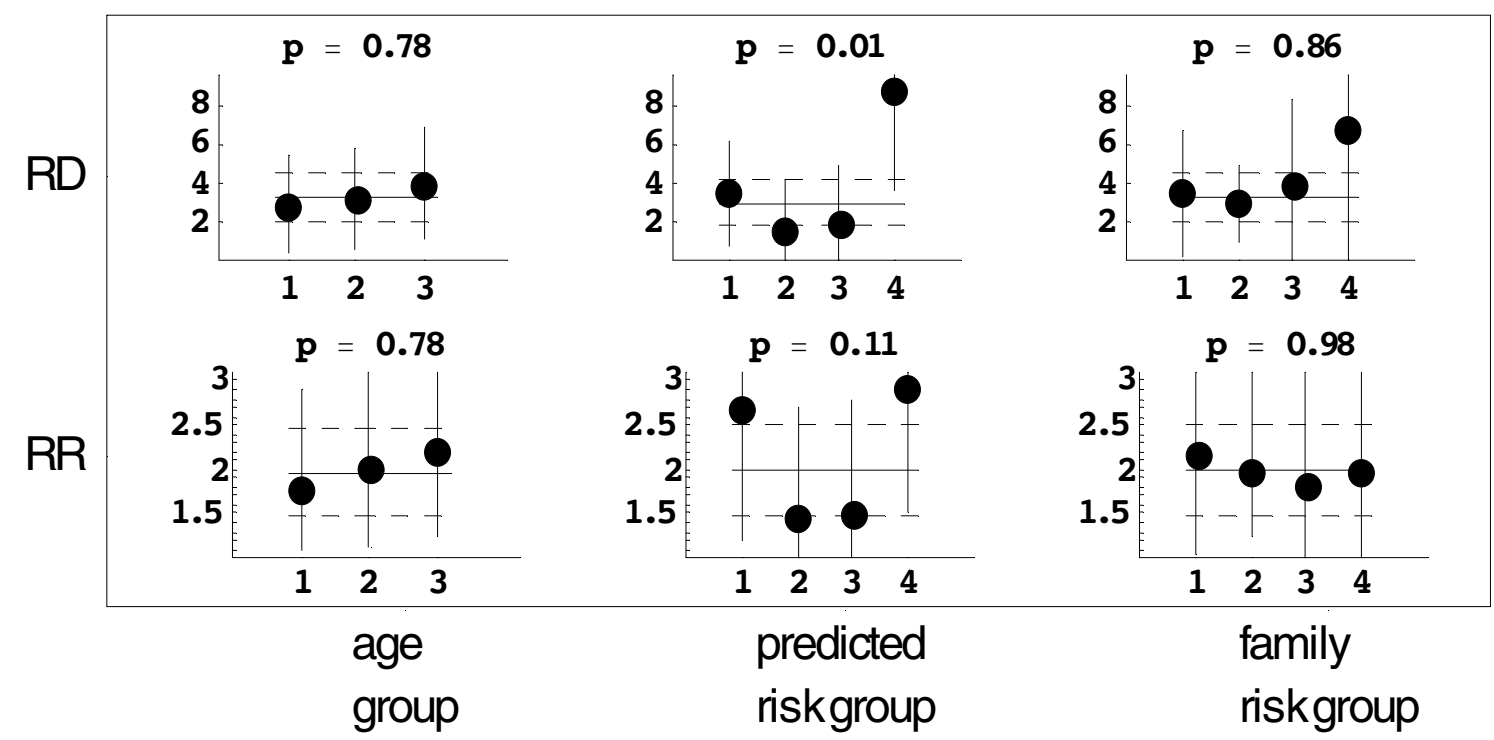

\section{Figure I}

Data from the tamoxifen prevention trial. See text for a description of groups. Horizontal lines are estimates and $95 \%$ confidence intervals for model for constant absolute risk difference per 1000 (RD) or relative risk (RR). P-values correspond to likelihood ratio tests comparing the models with varying and constant risk difference or relative risks.

However if Constant $R R$ is the true state of nature, the power will be greater in the high-risk group than the general population. Thus there is high probability that the power could be reduced when studying high-risk subjects than when studying the general population. Therefore, there is no free lunch in terms of lowering statistical power.

\section{Possibly increased costs}

Even if the model is correct (namely $p_{C}$ and $p_{I}$ are correctly chosen), the smaller trial of high-risk subjects may be more expensive than the larger trial of average-risk subjects from the general population. Consider the following two trials with a power of .90 and a one-sided type I error of .05. In the trial of high-risk subjects $p_{C}=.04$ and $p_{I}=.02$, and in the trial of average-risk subjects, $p_{C}=.02$ and $p_{I}=$ .01 . Suppose the statistic of interest is the absolute risk difference. To obtain sample size for each randomization group we use the standard sample size formula [4],

$n=\frac{\left(\sqrt{p(1-p)} 1.644485+\sqrt{p_{C}\left(1-p_{C}\right)+p_{I}\left(1-p_{I}\right)} 1.28155\right)^{2}}{\left(p_{C}-p_{I}\right)^{2}}$ where $p=\left(p_{C}+p_{I}\right) / 2,1.644485$ is the z-statistics corresponding to the 95 th percentile of the normal distribution (for a one-sided type I error of .05) and 1.28155 is the zstatistics corresponding to the 90th percentile (for a power of .90). Based on (4), the sample size for a trial using average-risk subjects from the general population study is 2529 per group and the sample size for a trial of high-risk subjects is 1244 per group. Let $C_{R}$ denote the cost of recruitment per subject and $C_{I}$ denote the cost of intervention and follow-up per subject averaged over the two randomization groups. Suppose high risk subjects comprise a fraction $f$ of the general population. The total cost of the trial for average-risk subjects from the general populations is

$C_{\text {general }}=2\left(C_{R} 2529+C_{I} 2529\right)$,

and the total cost of the trial for high-risk subjects is

$C_{\text {high-risk }}=2\left(C_{R} 1244 / f+C_{I} 1244\right)$.

where the factor of 2 is for the two randomization groups. The condition for the trial of high-risk subjects to cost 
more than the trial of average-risk subjects (namely $C_{\text {high- }}$ risk $>C_{\text {general }}$ ) is

$\frac{C_{R}}{C_{I}}>\frac{2529-1244}{1244 / f-2529}$,

when $1244 / f-2529>0$. If $f=.20$, the trial of high-risk subjects will cost more than the trial of average-risk subjects if $C_{R} / C_{I}>.34$. If $f=.10$, the trial of high-risk subjects will cost more than the trial of average-risk subjects if $C_{R} /$ $C_{I}>.13$.

In many cancer prevention trials the above values of $C_{R} / C_{I}$ are likely. For example, diagnostic testing to identify highrisk smokers can include expensive airway pulmonary function tests or bronchoscopy. In the future, more trials will likely involve expensive genetic testing of subjects [5] with costs ranging from $\$ 350$ to almost $\$ 3,000$ per test according to recent information from Myriad Genetic Laboratories. As part of a sensitivity analysis related to genetic testing of subjects prior to enrollment in a trial, Baker and Freedman [5] considered values of .1, .5, and 1 for ratios similar to $C_{R} / C_{I}$.

Even without diagnostic testing, the costs of obtaining high-risk subjects can be substantial. If $f=.10$, the initial recruitment will require ten times the number of people as for a trial of average-risk subjects from the general population. This increased recruitment would likely require higher advertising costs and increased overhead costs from the inclusion of additional institutions.

One additional consideration is how noncompliance and contamination affect the intent-to-treat analysis. If noncompliance and contamination can be anticipated, the investigator can correspondingly adjust the sample size and costs. Mathematically the effect of noncompliance and contamination is to change the values of $p_{C}$ and $p_{I}$ in (4), which would then affect (5) and (6). In some settings, investigators may anticipate that high-risk subjects are more likely to comply with the intervention than averagerisk subjects. To compensate for the anticipated increased compliance, study designers could reduce the sample size which would lower costs. However, in other situations, investigators may anticipate that subjects found to be at high-risk on a diagnostic test would likely seek the best therapy outside of the trial rather than chance randomization to standard or experimental therapy. To compensate for the anticipated dilution in treatment effect, investigators would need to increase the sample size which would increase the costs.

For the above reasons even if the probabilities under the alternative hypothesis are correctly specified, some trials of high-risk subjects may be more expensive than larger trials of average-risk subjects with the same power and type I error.

\section{Possibly misleading ratio of benefits to harms}

When there is strong evidence prior to the trial of a high probability of harmful side effects due to the intervention, one would want to restrict the intervention to high-risk subjects. Otherwise, some investigators may be tempted to estimate the ratio of benefit to harms in the trial of high-risk subjects and extrapolate the ratio to average risk subjects. Unfortunately, even if the assumption of constant relative risk over risk categories were true, extrapolating the benefit-harm ratio from a high risk group to the general population could be misleading.

Suppose that in a randomized trial involving average-risk subjects from the general population the probability of cancer is .02 in the control arm and .01 in the study arm. Also suppose that relative risk is same in the general population as in the high-risk group, so that in a randomized trial involving a high-risk group, the probability of cancer is .04 in the control arm and .02 in the study arm. Furthermore, suppose that the probability of harmful side effects is the same for high-risk subjects as for average-risk subjects in the general population, namely .015 in the control arm and .025 in the study arm. Based on these results, for every 1000 high-risk persons who receive the intervention, $(.04-.02) 1000=20$ will benefit from the intervention and (.025 - .015) $1000=10$ will be harmed by side effects, yielding a benefit-harm ratio of $20: 10=2: 1$. Similarly for every 1000 average-risk person who receive the intervention, $(.02-.01) 1000=10$ will benefit from the intervention and $(.025-.015) 1000=10$ will be harmed by side effects yielding a benefit-harm ratio of 10:10 =1:1. In this example it would be incorrect to extrapolate the high benefit-harm ratio estimated from the high-risk group to the general population for whom the benefitharm ratio is much lower. For many cancer prevention interventions, the ratio of life-threatening disease avoided to life threatening harms would be favorable in the highrisk group but not favorable when extrapolated to the general population.

\section{Conclusion}

There is no "free lunch" when using high-risk subjects in prevention trials design to make inference about the general population. Using high risk subjects instead of average-risk subjects from the general population may lower statistical power, increase costs, and yield a misleading ratio of benefit to harms than actually the case.

Given the substantial costs of definitive randomized trials in cancer prevention, and the importance of accurately assessing the balance of benefit and harm when treating healthy and asymptomatic people, it is therefore impor- 
tant to conduct trials in the actual target population rather than try to conduct them in high-risk populations with the plan to extrapolate results to the general population.

\section{Competing Interests}

The authors declare that they have no competing interests.

\section{Authors' contributions}

SGB wrote the initial draft, and BSK and DC made valuable improvements. All authors read and approved the final manuscript.

\section{Additional material}

\section{Additional File 1}

Appendix A, worked-out calculations of power.

Click here for file

[http://www.biomedcentral.com/content/supplementary/1471-

2288-4-24-S1.pdf]

\section{Additional File 2}

Appendix B, likelihood formulations

Click here for file

[http://www.biomedcentral.com/content/supplementary/1471-

2288-4-24-S2.pdf]

\section{References}

I. Lachin JM: Introduction to sample size determination and power analysis for clinical trials. Control Clin Trials I98I, 2:93-I I3.

2. Bach PB, Kattan MW, Thornquist MD, Kris MG, Tate RC, Barnett MJ, Hsieh LJ, Begg CB: Variations in Lung Cancer Risk Among Smokers. Journal of the National Cancer Institute 2003, 95:470-478.

3. Gail MH, Brinton LA, Byar DP, Corle DK, Green SB, Schairer C, Mulvihill J]: Projecting individualized probabilities of developing breast cancer for white females who are being examined annually. Journal of the National Cancer Institute 1989, 81:1879-I886.

4. Frideman LM, Furberg CD, DeMets DL: Fundamental of Clinical Trials John Wright: Boston; 198I.

5. Baker SG, Freedman LS: Potential impact of genetic testing on cancer prevention trials, using breast cancer as an example. Journal of the National Cancer Institute 1995, 87: I I 37- I | 44.

6. Fisher B, Costantino JP, Wickerham DL, Redmond CK, Kavanah M, Cronin WM, Vogel V, Robidoux A, Dimitrov N, Atkins J, Daly M, Wieand S, Tan-Chiu E, Ford L, Wolmark N: Tamoxifen for Pre vention of Breast Cancer: Report of the National Surgical Adjuvant Breast and Bowel Project P-I Study. Journal of the National Cancer Institute 1998, 90:137|-1388.

\section{Pre-publication history}

The pre-publication history for this paper can be accessed here:

http://www.biomedcentral.com/1471-2288/4/24/prepub
Publish with Biomed Central and every scientist can read your work free of charge

"BioMed Central will be the most significant development for disseminating the results of biomedical research in our lifetime. "

Sir Paul Nurse, Cancer Research UK

Your research papers will be:

- available free of charge to the entire biomedical community

- peer reviewed and published immediately upon acceptance

- cited in PubMed and archived on PubMed Central

- yours - you keep the copyright

Submit your manuscript here:

http://www.biomedcentral.com/info/publishing_adv.asp 